\title{
The Processes Which Cause the Appearance of Objects and Systems
}

\author{
Weitter Duckss \\ Independent Researcher, Zadar, Croatia
}

Email address:

wduckss@gmail.com

To cite this article:

Weitter Duckss. The Processes Which Cause the Appearance of Objects and Systems. American Journal of Astronomy and Astrophysics. Vol. 6, No. 3, 2018, pp. 72-80. doi: 10.11648/j.ajaa.20180603.13

Received: August 27, 2018; Accepted: September 30, 2018; Published: November 7, 2018

\begin{abstract}
The beginning of the formation of galaxies can be recognized in the planetary and stellar systems. The rotation speed of a galactic center determins the form of a galaxy an the ongoing processes. The forces of attraction and the rotation of stars firstly form binary systems. The objects that are locked down by their tidal forces or that posses an extremely slow rotation, i.e. they have no independent rotation - they don't have other objects orbiting around themselves; for example: Mercury, Venus and the majority of satellites. A very fast cyclone rotation (in an elliptical galaxy) creates huge friction, whichheats up matter; that can be seen on quasars and very fast-rotating small objects (stars) through the emission of radiation that takes place on the poles. A vast number of stars and other matter (the center of a galaxy), when rotating around the common center, act as a single body, related to the rest of the galaxy. A slow rotation of a galactic center (as in the stellar clusters) does not create a recognizable center (the center looks more like the ones of close binary systems), while the fast rotation creates the center that ranges from the northern to the southern pole of the center. The speed of rotation is not exclusively responsible for the size of an object (a galaxy, a star,...) because a fast rotation is a characteristic of both dwarf and giant galaxies. The same goes for a slow rotation. The same principle applies to stars. There are big stars with different speeds of rotation, and the same goes for small stars. There are hot stars with very small mass, but there are also hot giant stars. Cyclones (in the north and south poles of the galaxy nucleus) are responsible for acceleration and deceleration of galactical and stellar rotations (as well as the death of stars). The influx of hotter matter accelerates the rotation of an object (the influx of stars to the cyclone in the center of a galaxy).
\end{abstract}

Keywords: Effects of Rotation, Forming a Galaxy, Dark Matter, Light

\section{Introduction}

The goal of this article is to sum up the processes of the objects' formation in Universe, with a special review of galaxies. In this article, these basic laws of nature are used: a constant process of growth, valid for all objects in Universe [1]; matter attraction feature [2]; the effects of objects' rotation around their axes [3] and inside a system; a decrease of radiation intensity and temperature with the increase of distance from a source of radiation or temperature (an object that creates and emits radiation) [4]; the absence of light in Universe; a short debate on dark matter from the other angle [5]. I consider the rotation of objects as the central process which creates the systems of stars, galaxies, the clusters of galaxies, Universe, Multiverse,...; it creates all systems, determines their appearance and, related to stars, their temperatures, radii, colors, orbital speeds of the objects around a star, their numbers, asteroid belts and gas disks. [6]

\section{The Effects of Rotation Around an Axis (Objects) and a Center (Systems)}

\subsection{The Formation of a System by Rotation}

The observation of the rotational effects can be done through the orbits of objects around a central object. All orbits (of an object) are placed around the equatorial region or cut through it if they are inclined, i.e., if there is an inclination from the equatorial plane. The speed of an object that approaches a central object has nothing to do with the 
appearance of the orbit, because if it did, we would have had orbits around the poles [7]. The objects that lack an independent rotation (i.e., the objects that are tidally locked) or have an extremely slow rotation have no possibility to take and hold other objects in their own orbits (for example, Venus, Mercury, internal satellites (tidally locked).

Quote: These objects also have a speed, just as the objects that approach straight or with an inclination towards the equator do, but these speeds neither create orbits (new evidence, confirmation $[8,9]$ ), nor there are observations to support such claims. If there is no rotation, there is also no orbit, no matter what the speed of the incoming object is. end quote

The objects on their poles have no rotation related to the vertically incoming objects, therefore their collisions are almost the only option.

Quote: One object becomes a nova and a large number (millions) of others with the same parameters just go on the same way. It is necessary to consider some very rare factors, like, for example, the impacts of large objects into planets, but even more rare - those that hit only a small part of the objects (one event in more than ten million of objects - stars).

Within the growth of an object, some smaller object is starting a reaction when colliding with a star. If that should remain a rare event, it needs to be a specific event under the specific conditions. The only possible specificity is for that object (the errant objects, incoming from outside the Solar system) to arrive vertically onto one of the poles and to hit the opening of a cyclone that exists on the poles of stars. That way, it would get an opportunity to break into the interior of an object.

When discussing the vertical trajectories, it is necessary to point out that only the forces of attraction exist there, because an object creates the forces of repulsion in the horizontal direction only. end quote [10]
A part of an object goes through a central object, due to a constant movement of a central object (Sun $220 \mathrm{~km} / \mathrm{s}$ ) and goes irreversibly further into space.

\subsection{The Effects of the Stars' Speed of Rotation}

A star's speed of rotation causes its temperature (its temperature only partially depends on the mass of a star), its radius (ratio: the mass of a star / the radius of a star; Sun $=1$ ), surface gravity and the color of a star. The stars with a slow rotation are "cold" stars (with the exclusion of binary systems effects), independently of the mass of a star and its radius. Their color is red and they are dominant in Universe

(M type of stars, $0.08-0.45$ masses of Sun; $\leq 0.7$ R of Sun; $2,400-3,700^{\circ} \mathrm{K} ; 76,45 \%$ of the total number of stars in Milky Way (Harvard spectral classification);

all red stars above $0,45 \mathrm{M}$ of Sun are also included here, as well as the largest red (and other) stars in our galaxy). The stars with fast and very fast rotations are mostly present in nebulae, i.e., in the space which is rich with matter. Their total quantity in Milky Way makes 3,85\% (O class $\sim 0,00003 \%$ ). [11]

A radius, related to mass ( $\operatorname{Sun}=1$ ) is negative, when stars with a fast rotation are the subject matter, while it is completely opposite with cold, red, slowly-rotating stars. [12]

A bit of a remark: the author of this article disagrees with the current estimates of the stars' mass, as he claims they are the product of old hypotheses which lacked enough evidence to support them. The author suggests that a radius be equal to a mass when discussing slowly-rotating stars and that the mass decrease up to $100 \%$ with fast-rotating stars. For example, Melnick 42, 21,1 R of Sun, its mass should be around $30 \mathrm{M}$ of Sun (currently, $189 \mathrm{M}$ of Sun).

That would give the option to avoid these illogicalities:

Table 1. Star, type / mass / temperature.

\begin{tabular}{lllll}
\hline & Star & Type & Mass Sun=1 & Temperature ${ }^{\circ} \mathbf{K}$ \\
\hline 1. & WR 2, & WN4-s & 16 & 141.000 \\
2. & H Columbae & O & 16 & 33.000 \\
3. & Deneb & A & 19 & 8.525 \\
3. & Gamma Cassiopeiae & B & 17 & 25.000 \\
4. & VY Canis Majoris & M & 17 & 3.490 \\
5. & DH Tauri b & Planet; dist. 330 AU & 12 M Jupiter & 2.750 \\
6. & HIP 78530 b & Planet; dist. 740 AU & 24 M Jup. & $2.700(2.800)$ \\
7. & NML Cygni & M & 50 & 3.834 \\
\hline
\end{tabular}

Table 1. Stars, similar mass (except No 5, 6, 7), different classes (type) and temperatures.

A same or similar mass should produce the same or similar outcome, given other conditions are the same. These days, scientific community totally undervalues the rotation of objects and its effects.

Table 2. Stars, temperature/rotation speed/surface gravity, mass/radius.

\begin{tabular}{|c|c|c|c|c|c|}
\hline & Star & Mass, Sun 1 & Radius, Sun 1 & Temperature $^{\circ} \mathrm{K}$ & Rotation speed km/s \\
\hline 1. & $\begin{array}{l}\text { Stars with slow rotation } \\
\text { Arcturus }\end{array}$ & 1,08 & 25,4 & 4.286 & 2,4 \\
\hline 2. & R Doradus & 1,2 & $370 \pm 50$ & 2.740 & 340 day \\
\hline 4. & Kappa Persei & 1,5 & 9 & 4.857 & 3 \\
\hline 5. & Aldebaran & 1,5 & 44,2 & 3.910 & 634 day \\
\hline
\end{tabular}




\begin{tabular}{|c|c|c|c|c|c|}
\hline & Star & Mass, Sun 1 & Radius, Sun 1 & Temperature $^{\circ} \mathrm{K}$ & Rotation speed km/s \\
\hline 6. & Hamal & 1,5 & 14,9 & 4.480 & 3,44 \\
\hline 7. & Iota Draconis & 1,82 & 11,99 & 4.545 & 1,5 \\
\hline 8. & Pollux & 2,04 & 8,8 & 4.666 & 2,8 \\
\hline 9. & Beta Ursae Minoris & 2,2 & 42,6 & 4.030 & 8 \\
\hline 10. & Beta Andromedae & $3-4$ & 100 & 3.842 & 7,2 \\
\hline 11. & $\begin{array}{l}\text { Betelgeuse } \\
\text { Fast-rotating stars }\end{array}$ & 11,6 & $887 \pm 203$ & 3.590 & 5 \\
\hline 12. & IK Pegasi & 1,65 & 1,6 & $7.000 / 35.000$ & $<32,5$ \\
\hline 13. & Alpha Pegasi & 4,72 & 3,51 & 9.765 & 125 \\
\hline 14. & $\eta$ Aurigae & 5,4 & 3,25 & 17.201 & 95 \\
\hline 15. & Eta Ursae Majoris & 6,1 & 3,4 & 16.823 & 150 \\
\hline 16. & Spica secondary & 6,97 & 3,64 & 18.500 & 87 \\
\hline 17. & Spica secondary & 10,25 & 7,7 & 22.400 & 199 \\
\hline 18. & Gamma Cassiopeiae & 17 & 10 & 25.000 & 432 \\
\hline 19. & WR 102 & 19 & 0,39 & 210.000 & 120 \\
\hline 20. & Zeta Puppis & $22,5-56,6$ & $14-26$ & $40.000-44.000$ & 220 \\
\hline 21. & S Monocerotis & 29,1 & 9,9 & 38.500 & 120 \\
\hline 22. & Alnilam & $30-64,5$ & $28,6-42$ & 27.000 & $40-70$ \\
\hline 23. & Alnitak Aa & $33 \pm 10$ & $20.0 \pm 3.2$ & 29.000 & $110 \pm 10$ \\
\hline 24. & HD $5980 \mathrm{C}$ & 34 & 24 & 34.000 & 120 \\
\hline 25. & HD 5980 A & 61 & 24 & 45.000 & 250 \\
\hline 26. & HD 93250 & 83,3 & 15,9 & 46.000 & 130 \\
\hline 27. & HD 269810 & 130 & 18 & 52.500 & 173 \\
\hline 28. & VFTS 682 & 150 & 22 & $52.200 \pm 2.500$ & 200 \\
\hline 29. & Melnick 42 & 189 & 21,1 & 47.300 & 240 \\
\hline 30. & R136a2 & 195 & 23,4 & 53.000 & 200 \\
\hline
\end{tabular}

Table 2. Stars, relationship: temperature/rotation speed/ surface gravity and mass/radius. No 1-12 cold stars, 13-29 hot stars.

The influence of rotation is more significant with stars that possess larger mass, because warming up and pressure are the result of friction, occurring between layers of a star. These stars that rotate faster will have higher temperatures than small stars, with the same or slower rotation (binary effects excluded).

Slowly-rotating stars have less significant surface gravity than the fast-rotating stars. [12]

Table 3. Stars, temperature/rotation speed/surface gravity; mass/radijus.

\begin{tabular}{|c|c|c|c|c|c|c|}
\hline & Star & Temperature ${ }^{\circ} \mathbf{K}$ & Rotation $\mathrm{km} / \mathrm{s}$ or day & Mass, Sun 1 & Radijus, Sun 1 & Surface gravity cgs \\
\hline 1. & Betelgeuse & $3.140-3641$ & 5 & $7,7-20$ & $950-1200$ & 0,5 \\
\hline 2. & Aldebaran & 3.910 & $643 \mathrm{~d}$ & $1,5 \pm 0,3$ & $44,2 \pm 0,9$ & 1,59 \\
\hline 3. & Pollux & $4.666 \pm 95$ & $558 \mathrm{~d}$ & $2.04 \pm 0,3$ & $8.8 \pm 0,1$ & 2,685 \\
\hline 4. & Polaris & 6.015 & $119 \mathrm{~d}$ & 4,5 & $46 \pm 3$ & 2.2 \\
\hline 5. & Canopus & 7.350 & 8,0 & $9,0-10,6$ & $71,4 \pm 4,0$ & 2,1 \\
\hline 6. & Beta Pictoris & $8.052(9.790)$ & 130 & 1,75 & 1,8 & 4,15 \\
\hline 7. & Denebola & 8.500 & 128 & 1,78 & 1.728 & 4,0 \\
\hline 8. & Fomalhaut & 8.590 & 93 & 1,92 & 1,842 & 4,21 \\
\hline 9. & Vega & $9.692 \pm 180$ & $12,5 \mathrm{~h}$ & 2,135 & $2,36 \times 2,81$ & 4,1 \\
\hline 10. & Sirijus a & 9.940 & $225-250$ & 2.02 & 1,711 & 4,33 \\
\hline 11. & Albireo B & $13.200 \pm 600$ & 0,6 days & 3,7 & 2,7 & 4,00 \\
\hline 12. & Sirijus b & 25.200 & 1 & 0,978 & 0,0084 & 8,57 \\
\hline
\end{tabular}

Table 3. Stars, No 1-7 low temperatures, small rotation speed, small surface gravity, in relation: radius>mass; No 8-16 high temperature, high Surface gravity, in relationship: radius $<$ mass (Sun $=1$ ).

\subsection{Gravitationally Bound Objects}

Gravity and rotation create systems. Super clusters of galaxies are the largest gravitationally-bound objects known today. The rotation of a cluster is different from zero. [13]

Table 4. Galaxy, distance /speed.

\begin{tabular}{llll}
\hline & Galaxy & Distance Mly & Red shift km/s \\
\hline 1. & NGC 4450 & $\sim 50$ & $1954 \pm 4$ \\
2. & NGC 4262 & 50,0 & $1359 \pm 4$ \\
3. & NGC 4550 & 50.0 & $381 \pm 9$ \\
4. & Messier 89 & $50 \pm 3$ & $290 \pm 5$ \\
5. & NGC 4435 & 52 & $0.002638(\mathrm{z})$ \\
6. & Messier 86 & $52 \pm 3$ & $-244 \pm 5$ \\
\hline
\end{tabular}




\begin{tabular}{llll}
\hline & Galaxy & Distance Mly & Red shift $\mathbf{~ k m} / \mathbf{s}$ \\
\hline 7. & Messier 61 & $52.5 \pm 2.3$ & $1483 \pm 4$ \\
8. & Messier 91 & $63 \pm 16$ & $486 \pm 4$ \\
9. & NGC 4388 & $65.10 \pm 18.43$ & 2.524 \\
\hline
\end{tabular}

Table 4. Galaxy, relationship: distance 50-65.10 $18.43 \mathrm{Mly} /$ speed of movement.

Table 5. Supercluster, galactical clusters, galaxy, redsfift/distance.

\begin{tabular}{llll}
\hline & Supercluster (galaxy) & Redsfift (z) & Distance M ly \\
\hline 1 & The Laniakea Supercluster & $+0,0708$ & 250 \\
2 & Horologium Supercluster & 0,063 & 700 \\
3 & Abell 754 & 0,0542 & 760 \\
4 & Abell 133 & 0,0566 & 763 \\
5 & Corona Borealis Supercluster & 0,07 & 946 \\
6 & CID-42 & 0,359 & $3.900(3,9$ Gly) \\
7 & Saraswati Supercluster & 0,28 & 4.000 \\
8 & Einstein Cross & 1,695 & 8.000 \\
9 & Twin Quasar & 1,413 & 8.700 \\
10 & Lynx Supercluster & $1,26 \& 1,27$ & 12.900 \\
\hline
\end{tabular}

Table 5. The Universe, Supercluster, galactical clusters, galaxy: redsfift (z)/distance M ly(G ly).

Table 6. Galaxies, redsfift/distance/speed.

\begin{tabular}{lllll}
\hline & galaxies & Redsfift (z) & Distance billion ly & Speed Km/s \\
\hline 1 & EQ J100054+023435 & 4.547 & 12,2 & 280.919 \\
2 & Q0906 +6930 & 5,47 & 12,3 & 299,792 \\
3 & Z8 GND 5296 & $7,5078 \pm 0,0004$ & 13,1 & $291.622 \pm 120$ \\
4 & GN-z11 & 11,09 & 13,4 & $295.050 \pm 119.917$ \\
\hline
\end{tabular}

Table 6. The Universe, relationship: redsfift (z)/distance G ly/speed km/s.

Besides rotation, there is also the law of (matter) attraction, which causes collisions, larger and smaller fusions of galactical clusters and Supercluster. [14] One should make a distinction between collisions, in which the orbits of objects or systems are different, and fusions, in which objects share the same orbit and gravity causes a soft fusion of objects (for example, 67P/Churyumov-Gerasimenko).

The accumulation is a constant growing process from the formation of particles, the accumulation of particles into nebulae,..., joining into (chemical) compounds, the formation of smaller and larger objects. Stars, star systems, binary stars which are the initial stage of the formation of star clusters, galaxies, galactical clusters and finally Universe are all created with the increase in mass and in the force of pressure (which depends on the speed of rotation). A part of matter gets disintegrated by the explosions of stars. These explosions cause even or more significant results than those, made by the collisions in LHC in Switzerland. [15]

Quote: Despite destruction (the disintegration of matter), the observations show that the Universe is not losing its mass. On the contrary, it increases. It means that the Universe is efficiently replacing all of the lost matter, the minimum of which is 20 quadrillion of the Sun's masses, and even "some" more.

It is not to be forgotten that a smaller part of matter is also been disintegrated in the collisions of waves and particles. In order for the muons to be registered at all in the laboratories, a countless number of particle disintegrations needs to occur. It is an everlasting occurrence on the objects orbiting around a star from the beginning of time till these days and until a star becomes a nova. A good portion of matter is being disintegrated in the collisions of objects and galaxies.
Therefore, the colossal dimensions are not related only to the creation of matter, but also to the growth of all objects within stellar systems, galaxies and the Universe. Millions of craters are only a reminder of that process being contiguous and ongoing. end quote [16]

The author of the article discusses the following two or at the most three wholes (Multiverse,...), based on the decrease of temperature and radiation intensity with the increase of distance from the source, on the constant growth of gravitationally related systems, on systems behaving as a single object in attracting matter, inside the space in which the temperature is $0^{\circ} \mathrm{K}$ and the processes are still or extremely slow. [17]

An object in an orbit can approach or distance itself from a central object. It depends on the influx of matter to the object. If an object in an orbit has a relatively low influx of matter from a central object, it starts falling slowly to the central object (Mars/ Phobos) and the process is opposite when the influx of matter is more significant on the object in the orbit - it starts moving away (Earth/ Moon) (similar to the relation of a pendulum and a weight). The same situation is with the systems, with a remark that a faster rotation accelerates the processes.

The law of low temperatures is manifested in star systems and galaxies; the objects have higher orbital speeds with lesser gravitational effects. The temperature, which is below the melting point of helium, $4,216^{\circ} \mathrm{K}$, is responsible for it. The stars that are on the edges of galaxies, just as the objects on the edges of star systems, have higher speeds with lesser gravitational effects than their neighboring objects that are closer to the center. [18] 


\subsection{The Formation of Galaxies}

Matter attraction gathers objects into systems and rotation regulates these systems. When a large number of stars rotate around the common center in a relatively small volume (i.e., in the centers of galaxies), they act as a single object and create systems similar to star systems. A galactical disk is created on the same principles as the orbits of objects around stars and asteroid belts or gas disks; rotation, the speed of rotation, the force of attraction. [19] In a large majority of situations, central objects represent almost the whole mass of a system (Sun $99,86 \%$ ).

There are different galactical centers inside the general process of growth. Slow rotations create centers made of stars and other matter that look like the spherical groups of stars (there is a big difference in the speed of rotation) and they do not create a familiar-looking center inside the galactical center. [20] Cyclones, that break down a large part of stars and create a completely new and the largest object in Universe, are formed by fast rotation on the poles of the galactical centers. [21]

The speed of rotation is not exclusively responsible for the size of an object (a galaxy, a star,...) because a fast rotation is a characteristic of both dwarf and giant galaxies. The same goes for a slow rotation. The same principle applies to stars. There are big stars with different speeds of rotation, and the same goes for small stars. There are hot stars with very small mass, but there are also hot giant stars. The same applies to cold stars and those stars, which temperatures are somewhere in between.

Table 7. Galaxies, type / rotational speed.

\begin{tabular}{llll}
\hline \multicolumn{2}{c}{ galaxies } & type galaxies & Speed of galaxies \\
\hline Fast-rotating galaxies & & "X-ray observations of RX J1131-1231 (RX J1131 for short) show it is \\
1 & RX J1131-1231 & quasar & whizzing around at almost half the speed of light. [22] [23]" \\
& "possess a significant amount of rotation around the major axis" \\
2 & Spindle galaxy & elliptical galaxy & Within the knot, the rotation measure is 40 \pm 8 rad m-2 [24] \\
Contrary to: Slow Rotation & Lenticular Galaxy & \\
4 & Andromeda Galaxy & spiral galaxy & maximum value of 225 kilometers per second \\
5 & UGC 12591 & spiral galaxy & the highest known rotational speed of about 500 km/s, \\
6 & Milky Way & spiral galaxy & $210 \pm 10$ (220 kilometers per second Sun) \\
\hline
\end{tabular}

Table 7. galaxies, relationship: type galaxies / rotational speed of galaxies; No 1-3 Fast-rotating galaxies, No 4-6 Slow-rotating galaxies.

The speed of rotation affects the form of a galaxy and more dynamic processes inside such galaxies.

Table 8. Galaxies, type/ size.

\begin{tabular}{llll}
\hline & galaxies & type of galaxies & speed of galaxies \\
\hline Large galaxies & (fast-rotating) & & \\
1 & APM $08279+5255$ & elliptical galaxy & giant elliptical galaxy [25] \\
2 & Q0906 +6930 & blazar & the most distant known blazar \\
3 & OJ 287 & BL Lacertae object & the largest known objects \\
4 & S5 $0014+81$ & blazar & giant elliptical galaxy \\
5 & H1821 +643 & quasar & the most massive black hole \\
Contrary to: Dwarf galaxies (fast-rotating) & & \\
6 & Messier 110 & elliptical galaxy & dwarf elliptical galaxy \\
7 & Messier 32 & "early-type" & dwarf "early-type" galaxy \\
8 & NGC 147 & spheroidal galaxy & dwarf spheroidal galaxy \\
9 & NGC 185 & spheroidal galaxy & dwarf spheroidal galaxy \\
\hline
\end{tabular}

Table 8. galaxies, relationship: type of galaxies/ size of galaxies; No. 1-5 Large galaxies (fast-rotating), No. 6-9 Dwarf galaxies (fast-rotating).

\subsection{Changing the Structure of Galaxy, the Increase of Radiation Intensity with the Increase of the Speed of Rotation}

With the increase of speed of rotation (including faster orbits of stars and changing the structure in the centers of galaxies) there is also the increase of intensity and quantity of radiation coming from the openings of a cyclone on the poles of a central structure of our galaxy.

If the diameters of a galactical central object are estimated to be a few tens of thousands of light-years, the nature of the Milky Way's bar is actively debated, with estimates for its half-length and orientation spanning from 1 to $5 \mathrm{kpc}(3,000$ 16,000 ly [26] or 40 thousand ly on the equator and 30 thousands ly (according to some other sources [27] ) from a pole to the other one. It's diameter: the size of a supermassive black hole is $\sim 0,001-400$ AU [28] - there is a disparity between a central point (a black hole should be there) and a pole of the central structure of a galaxy (different occurrences and the beginning of different radiation emissions are measured there). The distance from the horizons (poles) and the center is 1.500 to $15.000 \mathrm{ly}$ and more, when giant galaxies with a very fast rotation are discussed.

The emissions of radiation are measured on the poles that are 3.000 to 30.000 ly and more far from each other and that proves the existence of cyclones (cyclones and whirls on stars [29]). Cyclones (the eye of a cyclone) are the places of 
occurrence for all occurrences that have ever been measured (radiation emissions, star formations, etc.). Their existence have been confirmed on the poles of Sun, Jupiter, Saturn, etc. They are formed due to the rotation of an object - and galaxies, especially their centers, rotate.

\subsection{Cyclones, Acceleration of Galaxies, the Increase of the Intensity of Radiation, Due to the Increase in Rotation}

Cyclones are responsible for acceleration and deceleration of galactical and stellar rotations (as well as the death of stars [10]).

The influx of hotter matter accelerates the rotation of an object (the influx of stars to the cyclone in the center of a galaxy; related to stars, objects heat up by passing through the atmosphere and photosphere of a star [29] ). It is known that hot and fast-rotating stars are mostly found in nebulae or other matter-enriched space. Recent appearances of the objects from the outside of our system, A / 2017 U1 [30] (1I / 2017 U1) [31] (inclination 122.69 ${ }^{\circ}$ ) and C/2012 S1 [32] (inclination $62,4^{\circ}$ ) confirm that such events are no rarity even in the space, which is less matter-enriched.

The size of a galaxy (as well as stars) depends on the quantity of matter in the space around it (free stars, the clusters of stars, smaller and larger galaxies with or without a central structure, nebulae, etc.). Galaxies with a faster rotation experience stronger attraction forces and also the possibility to grow faster. That fact alligns them with the galaxies that are younger than those with a slower rotation if there are similar masses or sizes and similar quantities of matter in their space. The same goes for the stars; the stars with a faster rotation grow faster - if other factors are similar. These similarities are present in our system, too, and are related to the planets with a faster rotation.

The formation of objects and galaxies occurs in a very cold space (the temperatures of $2-3^{\circ} \mathrm{K}$ ), it supports superconductivity (radiation expands at the speed of $\sim 300.000 \mathrm{~km} / \mathrm{s}$ ), in space, waves and radiation lose their intensity with the growing distance. The temperatures below $4,216^{\circ} \mathrm{K}$ (below the boiling point of helium) make it possible for the objects in that zone to move faster - if other conditions are similar - unlike the objects which temperatures are above $4,216^{\circ} \mathrm{K}$. At galaxies and stars, these things happen on the edges of these systems, where the results of measuring the speed of objects indicate faster movement than of those objects, which are closer to the center of a system (The proof is accelerating Voyagers).

\section{Dark Matter in Space}

\subsection{Dark Matter}

I give evidence for the connection of dark matter existence by the processes that are visible and measurable inside our system. If a part of space is (almost) empty, without the presence of matter (dark matter), there should exist the following: an even spreading of Sun radiation and independence of the temperature increase due to radiation.
The temperature of space can be observed indirectly. The easiest way to gain the result is to observe the temperature on the dark side of an object (the minimal temperatures).

Table 9. Sun system, temperature deviation, temperatures/ distance.

\begin{tabular}{llll}
\hline & $\begin{array}{l}\text { The body in orbit } \\
\text { around the Sun }\end{array}$ & $\begin{array}{l}\text { minimum } \\
\text { temperatures }\end{array}$ & $\begin{array}{l}\text { Distance from the } \\
\text { Sun AU }\end{array}$ \\
\hline 1 & Mercury & $80(100$ equator) & 0,39 \\
2 & Moon & 100 & 1 \\
3 & Mars & 143 & 1.52 \\
4 & Vesta & 85 & 2,36 \\
5. & Ceres & 168 & 2,77 \\
6 & 67P/Churyumov- & 180 & 3,46 \\
7 & Gerasimenko & 70 & 5,20 \\
8 & Ganymede & $80 \pm 5$ & 5.20 \\
9 & Callisto & 38 & 30,11 \\
10 & Triton & 33 & 39,48 \\
\hline
\end{tabular}

Table 19. Sun system, temperature deviation, relationship: minimum temperatures ${ }^{\circ} \mathrm{K} /$ distance from the Sun AU.

These measurements of minimal temperatures show deviations from the accepted claims that the intensity of ("termal") radiation decreases with the square distance. Except Mars and Pluto, not all objects have enough quantity of atmosphere, which could cause doubt about the correct way of selecting objects in the example. If a factor of measurement imprecision is also taken into consideration, the deviations are still impossible to be removed as they show that the objects from the examples $1-5,20$ AU have the same or higher minimal temperatures than Mercury and they are also of the lesser or similar mass. Mercury and Ceres are in a group of objects, which are explored equally well and in details; however, it is shown that the minimal temperature on Ceres is two times higher, even though it should be decreasing, according to the law of radiation intensity decrease with the increase of square distance.

If deviation is excluded and minimal temperatures are observed very roughly, it is obvious that there is a temperature decrease with the increase in distance: Mean Solar Irradiance $(\mathrm{W} / \mathrm{m} 2)$ on Mercury is 9.116,4, Earth 1.366,1, Jupiter 50,5, na Pluto 0,878. [33]

At the end of our system, the temperature is estimated at less than $4^{\circ} \mathrm{K}$.

The decrease of radiation intensity is (visually) the most notable when measuring the radiation of stars. The further the objects, the lower the intensity (with regards and correction of mass and temperature of a star). An example of deviation can also be found in the termosphere of our planet (although that example is (partially) solved in the way that a certain quantity of radiation, allocated to a lesser quantity of particles, results with easier temperature rise to higher temperatures). The examples from the table eliminate the claims that radiation dissipate with the increase of space (67P/Churyumov-Gerasimenko is more than $3 \mathrm{AU}$ further from Mercury and its minimal temperature is by $100^{\circ} \mathrm{K}$ higher).

Quote: The existence of matter can be observed here, on Earth, too. A balloon, inflated 2-3 km deep under the water 
surface, will explode just before the surface or on it, due to the air expansion. The similar thing happens to the balloons, which are sent outside the atmosphere - they explode at the maximum altitude of 40 (104) km above the surface of Earth, due to the equalizing the pressures. There are different kinds of matter and different outcomes, but the final outcome is the same: the pressures get equalized. The balloons are moving in the direction, which is opposite to the activity of gravitation and they exclusively abide the law of equalizing the different pressures. The balloons "know" where is the less dense matter inside a volume. end quote [34]

The termal deviation and the decrease of temperature from a source to the edges of a system indicate that there is a similarity between some processes in space and in the atmosphere (of Earth). Due to the interaction of radiation with particles of atmosphere and object itself, matter warms up. Space also warms up, due to the activity of the same radiation and without visible matter being present. As radiation waves distance themselves from a source, the intensity of radiation decreases, as well as temperature (both minimal and maximal) of space and visible matter (an object). A similar example can be found on Earth. Water is the warmest on its surface. The lowest temperatures are in the deepest waters, if geological warmings are excluded (hot spots). Energy, different kinds of radiation and visible matter (which does not create its own warmth by geological processes) are very cold. The temperature of visible matter, when sources of radiation are not there at all or when they are too far, tends to be absolute zero $\left(0^{\circ} \mathrm{K}\right)$.

Space is the purest vacuum, but only if related to visible matter. According to evidence and definition, vacuum does not create friction which could reduce the intensity of radiation waves. A smaller part of particles in space, when collided by waves of radiation, turn into high-energy particles.

Quote: Different kinds of matter coexist one by the other and the transition from one into the other is more or less defined. That is impossible between matter and vacuum, because the pressures of matter and vacuum always tend to equalize and that is not what can be seen between the atmosphere and vacuum and with the gas (particle) gathering into nebulae, etc.

Right here, just outside (even inside) the atmosphere, there is the kind of matter, which is known to us, which had been defined and its influence on the visible matter calculated - it only remains to be detected. end quote [34]

If we push water out of a bowl, which is placed under water, it starts moving towards space with a lower pressure. The same thing happens to a balloon filled with helium.

\subsection{Light}

Light appears on the place of collision between radiation waves and particles. If there is no radiation, or if it is minimal, matter is very cold. If there is no visible matter, space warms up $\left(80\right.$ to $\left.180^{\circ} \mathrm{K}\right)$, just as visible matter. An important difference is that space does not produce light in collisions with radiation, no matter the intensity or sort of radiation

Warmth and light are produced only by visible matter. The light of Sun disappears immediately after leaving the atmosphere of Sun or with the disappearance of visible matter. Temperature drastically falls after leaving the atmosphere, but it does not disappear immediately (80 to $\left.100^{\circ} \mathrm{K}\right)$ - it gradually diminishes with the increase of distance through space. It does not matter, whether to name a space between a source and an object as invisible matter or just space. The important fact is that invisible space actively supports the processes that can be recognized in the visible mattter, too.

Space equals complete dark. Light appears only on objects (nebulae, planets, etc ). If there is no visible matter, there is no light. Stars (Sun - on the image) do not emit light, stars emit radiation. Light appearance and temperature growth occur in the collision of radiation and visible matter. There is no light immediately outside the atmosphere of Sun. [35]

\section{Conclusion}

Rotation and attracting matter create systems. Gravity without the effect of rotation, does not create systems.

The force of attraction (gravity) and the rotation of objects are basic preconditions to create dual or more complex systems (spherical and other groups of stars, galaxies and groups of galaxies). If gravity was the only existing or even dominating force, there would be no universe at all. Without the main creator of all systems - the rotation of objects, which places the falling objects into their orbits - the objects would fall vertically one upon the other. Rotation should not be observed only in the frame of a rotating object, but as a whole of an object and the space, with the attraction forces in it. Not only an object rotates, but the forces within its space rotate with it, too. [36] The rotation acts as antigravity. Due to the rotation, the antigravitational forces are changing the course of movement of the incoming objects from straight into round or ellyptic, around the bigger rotating object. In that way, the collapse of the minor part of that mass or these objects, existing in a new way, does not occur. [37] The rotation creates vortexes and cyclones (at the poles) in the center of galaxies and stars. Central objects in the centres of the galaxies observe more complex laws that are not based on the physical black holes. Beginning from the stars the size of our Sun, even the low speed rotations cause polar cyclones, which will in time turn into whirlwinds of the galactic size (up to 30000 light-years). They are able to hold together such a massive objects; the rotation of matter around a whirlwind holds the whole galaxy together. [38] Greater distance weakens the intensity (force) of waves (radiation). Lesser intensity of waves is registered as a greater shift into red. A very important fact needs to be stressed here: although after certain distance only red shift is registered, at the same time - on that and on all other distances - the collisions of galaxies are registered, or the blue shift between the objects in collision. There is an increase of speed along with the weakening of the intensity of waves, but by no means in 
numbers that are these days taken as an undeniable evidence. The rotation of the clusters of galaxies (speeds of movement by orbits) and the Universe (the rotation) is occurring many times slower. [39]

\section{Acknowledgements}

Madam Sylvie Wallimann-Crépin's Editorial Committee of EPD Sciences (2004) for the first boost at the beginning of the research.

Professor Zoran Ćoso, University of Zadar, for the translations in English and Russian.

My wife, Ranka Sedić, who funds this independent research.

\section{References}

[1] W. Duckss

http://www.globalscientificjournal.com/researchpaper/ZadarsTheory-of-the-Universe.pdf, "Constant process"

[2] W. Duckss https://www.ijser.org/onlineResearchPaperViewer.aspx?Theformation-of-particles-in-the-Universe.pdf 7/2018

[3] W. Duckss http://www.globalscientificjournal.com/researchpaper/Theinfluence-of-rotation-of-stars-on-their-radius-temperature.pdf

[4] W. Duckss.

https://www.academia.edu/16790589/The_causal_relation_of space_and_the_absence_of_light_in_Universe

[5] W. Duckss http://www.unexplainedmysteries.com/forum/topic/268345-why-is-the-universe-dark/

[6] W. Duckss.

https://www.academia.edu/19025940/Why_there_is_a_ring_a n_asteroid_belt_or_a_disk_around the_celestial_objects

[7] W. Duckss https://www.academia.edu/26326626/Weitter_Ducksss_Theor y_of_the_Universe "Rotation of an object"

[8] https://www.nasa.gov/feature/jpl/small-asteroid-or-cometvisits-from-beyond-the-solar-system Oct. 26, 2017 "Small Asteroid or Comet 'Visits' from Beyond the Solar System".

[9] https://www.nasa.gov/feature/solar-system-s-first-interstellarvisitor-dazzles-scientists Nov. 20, 2017 "Solar System's First Interstellar Visitor Dazzles Scientists".

[10] W. Duckss http://www.ijser.org/onlineResearchPaperViewer.aspx?Observ ation-of-the-Universe-through-questions.pdf "What are the dimensions of destruction and creation in the Universe?", Article No 7.

[11] W. Duckss

http://www.ijser.org/onlineResearchPaperViewer.aspx?Reasse ssment-of-the-old-but-still-employed-theories-of-Universethrough-database-checking.pdf Article No 2.

[12] W. Duckss http://www.ijser.org/onlineResearchPaperViewer.aspx?Theobservation-process-in-the-universe-through-the-database.pdf
The causal relation between a star and its temperature, gravity, radius and color Article No 1.

[13] W. Duckss

http://www.globalscientificjournal.com/researchpaper/DEMO LITION-HUBBLES-LAW-BIG-BANG-THE-BASIS-OFMODERN-AND-ECCLESIASTICAL-COSMOLOGY.pdf

[14] https://www.spacetelescope.org/static/archives/releases/scienc e_papers/heic1506a.pdf „The non-gravitational interactions of dark matter in colliding galaxy clusters" David Harvey1,2*, Richard Massey3, Thomas Kitching4, Andy Taylor2, Eric Tittley2

\section{[15] W. Duckss}

http://www.ijser.org/onlineResearchPaperViewer.aspx?Vacuu m-in-space-or-undetected-matter.pdf "Why did CERN fail?" Article No 3.

[16] W. Duckss

http://www.ijser.org/onlineResearchPaperViewer.aspx?Observ ation-of-the-Universe-through-questions.pdf "What are the dimensions of destruction and creation in the Universe?" Article No 7.

[17] W. Duckss https://www.svemir-ipaksevrti.com/the-Universerotating.html\#15b "Why is the Universe cold?".

[18] W. Duckss https://www.academia.edu/17760569/The_Oort_cloud._Speed _of_light_is_not_the_limit

[19] W. Duckss http://www.ijser.org/onlineResearchPaperViewer.aspx?Theobservation-process-in-the-universe-through-the-database.pdf "Why there is a ring, an asteroid belt or a disk around the celestial objects?" Article No 3.

[20] W. Duckss http://www.globalscientificjournal.com/researchpaper/Howare-the-spiral-and-other-types-of-galaxies-formed.pdf

[21] W. Duckss https://www.svemir-ipaksevrti.com/the-Universerotating.html\#14b "Observing the quasars through rotation"

[22] http://www.ijser.org/onlineResearchPaperViewer.aspx?Vacuu m-in-space-or-undetected-matter.pdf "The Reverse Influence of Cyclones to the Rotation of Stars" Article No 2.

[23] https://www.universetoday.com/110031/supermassive-blackholes-dizzying-spin-is-half-the-speed-of-light/ "Supermassive Black Hole's Dizzying Spin is Half the Speed of Light" Article written: 5 Mar, 2014Updated: 23 Dec, 2015 by Elizabeth Howell

[24] https://www.nasa.gov/press/2014/march/chandra-and-xmmnewton-provide-direct-measurement-of-distant-black-holesspin/\#.W4eeGugzaUl March 5, 2014 Release 14-069 "Chandra and XMM-Newton Provide Direct Measurement of Distant Black Hole's Spin"

[25] https://arxiv.org/abs/1707.05130 "CALIFA reveals Prolate Rotation in Massive Early-type Galaxies: A Polar Galaxy Merger Origin?" Athanasia Tsatsi, Mariya Lyubenova, Glenn van de Ven, Jiang Chang, J. Alfonso L. Aguerri, Jesús FalcónBarroso, Andrea V. Macciò (Submitted on 17 Jul 2017)

[26] https://en.wikipedia.org/wiki/APM_08279\%2B5255 APM $08279+5255$ etc

[27] https://en.wikipedia.org/wiki/Milky_Way\#Galactic_Center 
[28] https://en.wikipedia.org/wiki/Black_hole\#Physical_properties

[29] http://www.astrodigital.org/astronomy/milkywaygalaxy.html "The Milky Way Galaxy"

[30] W. Duckss http://www.ijser.org/onlineResearchPaperViewer.aspx?Weitter -Duckss-Theory-of-the-Universe.pdf "Functioning of the Universe"

[31] https://www.nasa.gov/feature/jpl/small-asteroid-or-cometvisits-from-beyond-the-solar-system Oct. 26, 2017 "Small Asteroid or Comet 'Visits' from Beyond the Solar System"

[32] https://www.nasa.gov/feature/solar-system-s-first-interstellarvisitor-dazzles-scientists Nov. 20, 2017, "Solar System's First Interstellar Visitor Dazzles Scientists"

[33] https://en.wikipedia.org/wiki/Comet_ISON\#Orbit the orbit of Comet ISON

[34] https://www.pveducation.org/pvedrom/properties-ofsunlight/solar-radiation-in-space "Solar Radiation in Space" Christiana Honsberg and Stuart Bowden
[35] W. Duckss http://www.ijser.org/onlineResearchPaperViewer.aspx?Vacuu m-in-space-or-undetected-matter.pdf Article No 1.

[36] W. Duckss https://www.academia.edu/37363821/Processes_in_Universe_ in_Pictures

[37] W. Duckss Https://www.academia.edu/11692363/Universe_and_rotation

[38] W. Duckss https://www.academia.edu/29645047/Universe2010.doc „The relations in the Universe“

[39] W. Duckss http://www.ijser.org/onlineResearchPaperViewer.aspx?Observ ation-of-the-Universe-through-questions.pdf "The forbidden article: Gravity and anti-gravity" Article No 4.

[40] W. Duckss http://www.ijser.org/onlineResearchPaperViewer.aspx?Observ ing-the-Universe-through-colors--blue-and-red-shift.pdf. 